\title{
The Association of Sexual Intercourse During Pregnancy With Labor Onset
}

\author{
Mahboobeh Kafaei Atrian ${ }^{1,2}$; Zohre Sadat ${ }^{3}$; Mahbobeh Rasolzadeh Bidgoly ${ }^{1}$; Fatemeh \\ Abbaszadeh ${ }^{1, *}$; Mohammad Asghari Jafarabadi ${ }^{4}$ \\ ${ }^{1}$ Department of Midwifery, Faculty of Nursing Midwifery, Kashan University of Medical Sciences, Kashan, IR Iran \\ 2 Department of Health Education, School of health-(International Campus), Iran University of Medical Sciences, Tehran, IR Iran \\ 3 Trauma Nursing Research Centre, Kashan University of Medical Sciences, Kashan, IR Iran \\ ${ }^{4}$ Road Traffic Injury Research Center, Tabriz University of Medical Sciences, Tabriz, Iran \\ ${ }^{*}$ Corresponding Author: Fatemeh Abbaszadeh, Department of Midwifery, Faculty of Nursing Midwifery, Kashan University of Medical Sciences, Kashan, IR Iran. Tel:+98-36155550021-5, \\ Fax: +98-36155556633, E-mail: abbaszadeh_f@kaums.ac.ir
}

Received: November 29, 2013; Revised: January 6, 2014; Accepted: February 1, 2014

\begin{abstract}
Background: Pregnancy is one of the most critical periods in women's lives. Sexual relationships change in this period. Monitoring of uterine contractions has been shown increase in uterine activity after sexual intercourse in pregnant women.

Objectives: This study aimed to determine the association of sexual intercourse during pregnancy with labor onset.

Patients and Methods: This cross-sectional study included 120 pregnant women with signs of labor onset at referral hospitals of Kashan University of Medical Sciences between November and March 2012. Signs of labor onset included labor pain, bloody show, or rupture of membrane. Subjects were investigated in two groups regarding history of coitus in the last week of pregnancy. Aquestionnaire containing demographic questions, obstetrical history, and sexual activity was completed by trained midwife through face-to-face interview. Chisquare test, Fisher's exact test, and t-test were used to check the homogeneity of the two groups for basic and confounding variables. Independent-samples t-test was used to compare differences between groups in terms of mean gestational age.

Results: There was no significant difference between groups in age $(P=0.434)$, body mass index $(P=0.705)$, neonatal weight $(P=0.421)$, maternal education $(\mathrm{P}=0.963)$, occupation $(\mathrm{P}=0.381)$, and parity $(\mathrm{P}=0.925)$. Gestational age at the time of delivery was significantly lower in intercourse group in comparison with control group based on last menstrual period $(\mathrm{P}=0.012)$ and ultrasonography $(\mathrm{P}=0.002)$. There was no correlation between intercourse and cause of admission $(\mathrm{P}=0.720)$. Type of delivery (cesarean section or vaginal delivery) was not affected by intercourse $(\mathrm{P}=0.820)$ or contact with semen $(\mathrm{P}=0.841)$. Results showed no significant difference in neonatal weight based on presence of sexual intercourse $(\mathrm{P}=0.422)$ or contact with semen $(\mathrm{P}=0.583)$ at the last week of pregnancy.

Conclusions: Sexual activity in last week of pregnancy might be associated with the onset of labor. Therefore, in the absence of complications in term pregnancy, sexual activity can be considered as a natural way to prevent post term pregnancy.
\end{abstract}

Keywords:Coitus; Pregnancy; Gestational Age; Labor Onset

\section{Background}

Pregnancy plays an important role in the sexual function and behaviors of women (1). Coital frequency and sexual desire decrease in most pregnant women, especially in the third trimester of pregnancy $(1,2)$. Sexual dysfunction can affect women's quality of life because decrease in sexual activity can adversely affect self-esteem and relationships of couples (1). Intercourse with breast/nipple stimulation cause oxytocin release, and orgasm lead to uterine contractions that can result to the labor (3). Monitoring of uterine contractions has shown an increase in uterine activity after intercourse in pregnant uterus (4). Sexual activity can accelerate the onset of labor, prevent prolonged pregnancy, and reduces the need of induction of labor $(4,5)$. Sexual activity during pregnancy improves the prognosis of labor and in the absence of contraindications, unprotected heterosexual intercourse is recommended in pregnant women (6).
On the other hand, other studies reported that gestational age would not be affected by coitus (7-9). Gestational age is higher in sexually active women than others (10). Orgasm was not associated with spontaneous labor and displayed a significant inverse association with spontaneous labor (8). Breast stimulation and sexual intercourse are ineffective methods for induction of labor or the assessments are insufficient to draw an evidencebased conclusion (9). Women who reported coitus were less likely to go into spontaneous labor prior to their scheduled labor induction $(8,11)$. There was no difference in Bishop Score between women who had sex in the preceding week and those who had not (10). An interventional trial reported that physician advice can increase reported coitus but the rate of spontaneous labor was not affected (7). Nonetheless, well-designed studies are required to determine the effect of sexual activity on labor onset at term pregnancies (8).

Copyright (C) 2015, Iranian Red Crescent Medical Journal. This is an open-access article distributed under the terms of the Creative Commons Attribution-NonCommercial 4.0 International License (http://creativecommons.org/licenses/by-nc/4.0/) which permits copy and redistribute the material just in noncommercial usages, provided the original work is properly cited. 


\section{Objectives}

Since many people felt women should have sex during pregnancy (12), physical and sexual activity in this period is more common than previously thought (13). In addition, given the contradictory studies in this field, the role of intercourse in inducing of labor is unknown (3). Therefore, this study was designed to determine the association of sexual intercourse during pregnancy with labor onset.

\section{Patients and Methods}

This study had a cross-sectional design. Research Council and Ethics Committee of Kashan University of Medical Sciences approved the study design (through letter No. 2978.1.5.29. C; date, Dec 20, 2011). Study was done at two hospitals, i.e. Shahid Beheshti and Shabih Khani Hospitals, in Kashan, Iran. Women who were hospitalized for delivery with signs of labor onset were enrolled in the study. Signs of onset of labor included labor pain, bloody show, or Rupture of Membranes (ROM). Physician on duty examined mothers and hospitalized them. Labor pain and bloody show were diagnosed based on history and progressive cervical dilatation. ROM, if not clearly visible, was diagnosed based on the results of fern test. Mothers were questioned whether they had intercourse in the last week of pregnancy. Then they were studied in two groups based on presence (group A) or absence (group B) of intercourse during the last week.

In the study of Tan et al. (5) the mean (SD) of gestational age for women with or without intercourse in the last month of pregnancy, were 39.9 (1.2) and 39.3 (1.1) weeks, respectively. Therefore, with 95\% confidence and $80 \%$ power, the sample size was calculated at 59 per group for comparison of means. Interviews were conducted while the women were hospitalized for labor onset, before discharge from hospital at admission room and postpartum ward. Initially, 175 women, 85 in group A and 90 in group $B$, were enrolled to the study. This sampling lasted from November to Marchs 2012. To assess the effect of contact with semen on gestational age, mothers who contacted with semen were compared with all mothers who had not, including those who had not intercourse at all. Questionnaire was completed by only one trained midwife through face-to-face interview. A two-stage sampling procedure was used. In the first stage, referral hospitals were selected and in the second stage, the cases were randomly selected to recruit in the study. Since these hospitals are referral hospitals in this issue, they cover a wide population of the Kashan city. Mothers with singleton pregnancy were included. Those with repeated or elective cesarean section, threatened abortion in the current pregnancy, uterine abnormalities, history of preterm labor or premature ROM (PROM), acute medical illnesses, preeclampsia, diabetes, or smoking, and those with inaccurate information were excluded. A checklist was used with three types of variables including: 1) demographic information including age, maternal education, and occupation; 2) obstetrical information including Last Menstrual Period (LMP), number of pregnancies, childbirth, abortion, and neonatal weight; and 3) main variables about gestational age and having intercourse in the last gestational week. The content validity of the questions was determined by a panel of ten expert people including those with MSc degree in midwifery, MSc degree in nursing and obstetricians. Because this checklist was record the facts, there was no need to assess reliability. Gestational age was calculated by two ways; one based on LMP and another one based on ultrasonography.

The subjects were free to participate in the study. They were assured that their information would remain confidential and signed an informed consent. Confidential interviews were conducted in a private environment. A digital glass scale (GS46, Germany) with 100 g accuracy was used to measure the weight in kilograms. A single non-elastic tape was used to measure the height in centimeters. Neonatal weight was measured in grams using baby Japanese analog scale. Reliability of scales was tested by 1-kg sinker. Reliability of the tape measure was evaluated using a wooden ruler, data were analyzed using SPSS 16 (SPSS Inc., Chicago, IL, USA). Kolmogorov-Smirnov test was used to investigate the distribution of data for quantitative variables. Chi-square test, Fisher's exact test, and Student's t-test were used to check the homogeneity of the two groups for basic and confounding variables. Independent-samples t-test was used to compare differences between groups in terms of mean gestational age. Confidence interval of $95 \%$ and significance level of 0.05 were considered.

\section{Results}

Initially, 175 people were interviewed among which 85 had intercourse during the last week before hospitalization and 90 had not. After follow up, nine cases in group A and 13 cases in group B were excluded because of incomplete or confusing information about gestational age. Moreover, 16 women in group A and 17 in group B were excluded because of inappropriate answer to questions. Finally, 60 persons in each group remained for the analysis. Figure 1 shows the loss of samples.

Table 1 shows that there was no significant difference between groups in age $(\mathrm{P}=0.434)$, body mass index $(\mathrm{P}=$ $0.705)$, neonatal weight $(\mathrm{P}=0.421)$, maternal education ( $\mathrm{P}$ $=0.963)$, and mother's occupation $(\mathrm{P}=0.381)$. The majority of people in both groups had completed high school and was house keepers. There was no significant difference between groups in terms of parity $(P=0.925)$, gravidity $(P=0.746)$, and abortion $(P=0.468)$. Most of the participants were nullipara (5.47\%). Results showed that intercourse $(P=0.820)$ or contact with semen $(P=0.841)$ had no effect on the type of delivery (cesarean section or vaginal delivery).

Table 2 shows that gestational age was significantly lower in intercourse group in comparison with control 
group based on LMP $(\mathrm{P}=0.012)$ and ultrasonography ( $\mathrm{P}$ $=0.002)$. Table 3 indicates that there was no correlation between intercourse and cause of admission to hospital $(P=0.720)$. Table 4 shows that gestational age was lower based on $\operatorname{LMP}(\mathrm{P}=0.005)$ and ultrasonography $(\mathrm{P}=0.001)$ in case of vaginal contact with semen. Results showed that mean (SD) of neonatal weight was 3074.57 (468.24) and 3134.16 (324.23) $\mathrm{g}$ in groups A and B, respectively. There was no significant difference between groups in neonatal weight based on history of intercourse at last week of pregnancy $(\mathrm{P}=0.422)$. Mean $(\mathrm{SD})$ of neonatal weight was 3081.48 (485.08) $\mathrm{g}$ in case of vaginal contact with semen and 3123.846 (318.70) g in case of no contact. This difference was not significant $(\mathrm{P}=0.583)$.

Table 1. Characteristics of the Subjects in Two Groups $(n=60)^{a, b, c}$

\begin{tabular}{lccc}
\hline Variables & Group A & Group B & P Value \\
\hline Maternal age, $\mathbf{y}$ & $25.61 \pm 4.54$ & $26.38 \pm 6.04$ & $0.434^{\mathrm{d}}$ \\
\hline BMI & $27.49 \pm 5.36$ & $26.46 \pm 3.86$ & $0.233^{\mathrm{d}}$ \\
Maternal education & & & $0.963^{\mathrm{e}}$ \\
$\quad$ Illiterate & $9(15.0)$ & $7(11.7)$ & $16(26.7)$ \\
\hline Elementary & $14(23.3)$ & $9(15.0)$ & $0.381^{\mathrm{e}}$ \\
\hline High school & $10(16.6)$ & $18(30.0)$ & $11(18.3)$ \\
\hline Diploma & $16(26.7)$ & & $53(88.3)$ \\
\hline Collegiate & $10(16.7)$ & $7(11.7)$ & \\
\hline Occupation & & & \\
\hline Housekeeper & $55(91.7)$ & $5(8.3)$ &
\end{tabular}

a Abbreviation: BMI, body mass index.

b Data are presented as Mean \pm SD or No. (\%).

c Presence (group A) or absence (group B) of intercourse in last week of pregnancy.

$\mathrm{d}$ Independent-samples t-test (equal variances were not assumed)

e Chi-square test.

Table 2. Comparison of Gestational Age Based on Days in Two Groups $(n=60)^{a, b}$

\begin{tabular}{lccc}
\hline Variable & Group A & Group B & LL-UL $^{\text {P Value }}$ \\
\hline GA based on LMP & $268.6 \pm 10$ & $273.12 \pm 9$ & $4.466(-7.923$ to -1.010$)$ \\
GA based on USG & $268.31 \pm 10$ & $273.7 \pm 8$ & $-5.466(-8.874$ to -2.058$)$ \\
\hline
\end{tabular}

a Abbreviations: GA, gestational age; LL-UL, lower limit to upper limit; LMP, last menstrual period; and USG, ultrasonography.

b Presence (group A) or absence (group B) of intercourse in last week of pregnancy.

${ }^{\mathrm{c}}$ Independent-samples t-test based on equal variances assumed (all Levene's test P values were > 0.05).

Table 3. Causes of Admission in Two Groups $(\mathrm{n}=60)^{\mathrm{a}, \mathrm{b}}$

\begin{tabular}{lccc}
\hline Cause of Admission & Group A & Group B & PValue C $^{\text {C }}$ \\
\hline Pain & $42(52.5)$ & $38(47.5)$ & 0.712 \\
Rupture of membranes & $16(45.7)$ & $19(54.3)$ & 0.712 \\
Bloody show & $2(40.0)$ & $3(60.0)$ & 0.712 \\
\hline
\end{tabular}

a Data are presented as No.(\%).

b Presence (group A) or absence (group B) of intercourse in last week of pregnancy.

${ }^{\mathrm{c}}$ Pearson's Chi-Square test (using exact Pvalue).

Table 4. Comparing Gestational Age Based on Days Regarding Vaginal Contact With Semen $(n=65)^{a, b}$

\begin{tabular}{lccc}
\hline Gestational Age & Group A $^{c}$ & Group B $^{\text {d }}$ & LL-UL $^{\text {P Value }^{\text {e }}}$ \\
\hline GA based on LMP & $268 \pm 10$ & $273 \pm 8$ & $-5.018(-8.432$ to -1.572$)$ \\
GA based on USG & $267 \pm 10$ & $273 \pm 8$ & $-6.067(-9.526$ to -2.607$)$ \\
\hline
\end{tabular}

\footnotetext{
a Abbreviations: GA, gestational age; LL-UL, lower limit to upper limit; LMP, last menstrual period; and USG, ultrasonography.

b Data are presented as Mean \pm SD or No. (\%).

C Vaginal contact with semen.

d No vaginal contact with semen.

e Independent-samples t-test based on equal variances not assumed (all Levene's test P values were $>0.05$ ).
} 


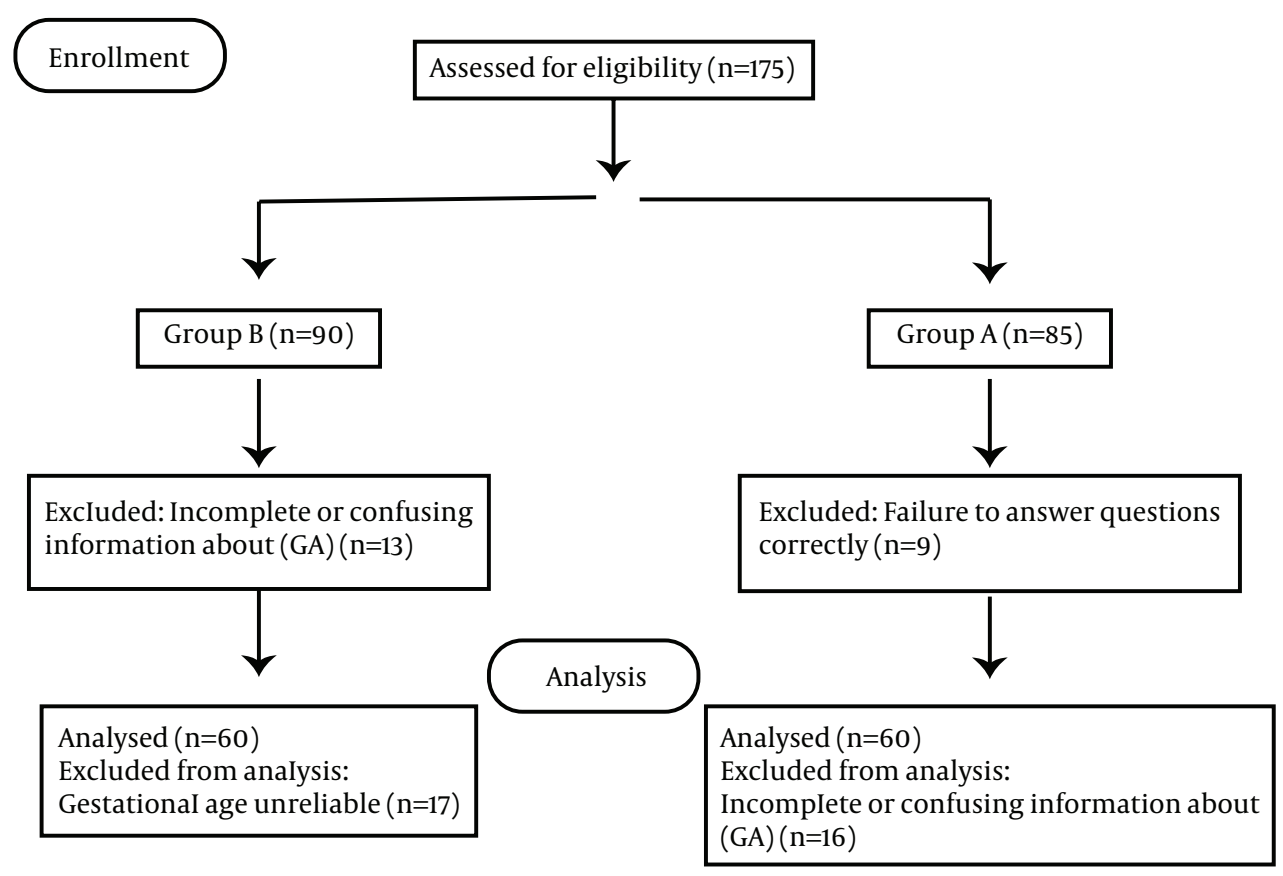

Figure 1. Flow Chart

\section{Discussion}

The aim of this study was to determine the association between intercourse during pregnancy and labor onset. Our results indicate that there was no significant difference between two groups in terms of maternal education and occupation. Nonetheless, Schaffir et al. showed that women with college education and professional career were more likely to have intercourse than others were (10). In this study, the most common complaints were consecutively pain, ROM, and bloody show. Ngwenya et al. showed that none of the patients who had been admitted with ROM had intercourse within 12 hours prior to the ROM (14). In this study, there was no correlation between intercourse and cause of admission to hospital. In a prospective cohort study on women declaring vaginal sexual intercourse after 37 weeks of pregnancy, Foumane et al. reported that sexually active women had a lower rate of caesarean sections and needed less oxytocin usage before expulsion (6).

In this study, the mean gestational age had significant difference between groups, which meant that gestational age based on LMP was significantly decreased with intercourse. Moreover, there was significant difference between groups based on ultrasonography, meaning that gestational age was lower in intercourse group. It has been recommended previously that a cervical ripening agent such as sexual intercourse can be used before labor induction (15). In a review study, Tenore (15) mentioned that sexual activity usually stimulates the breast and nipple, which can promote the release of oxytocin. With penetration, the lower uterine segment is stimulated. With penetration, the lower uterine segment is stimulated. This stimulation results in a local release of prostaglandins. Female orgasms have been shown to include uterine contractions sexually active would have significantly shorter active phase and second stage, a normal pattern of labor, and a higher rate of spontaneous deliveries (6).

In contrast, Kavanagh et al. in a review on clinical trial studies mentioned that the role of sexual intercourse as a method of induction of labor is uncertain. Sufficient power to detect clinically relevant differences in standard outcomes is needed to investigate intercourse as a method of induction (3). Schaffir et al. showed that gestational age at the time of delivery in women who were sexually active at term, was higher than those who were not but bishop scores had no significant difference between them (10). Human semen contains prostaglandins that are responsible for cervical ripening. In a prospective longitudinal study, Tan et al. reported that sexual intercourse was associated with reduction in post term pregnancy, duration of pregnancy, and induction of labor at 41 weeks (5). Foumane et al. (6) reported that sexual activity during pregnancy improves the prognosis of labor. They mentioned that most sexually active women were admitted during the active phase of labor, with the fetal head at station "zero" and concluded that when people do not feel any problem in pregnancy, sexual activity continues. On the other hand, those who experience complications such as cramps and pelvic pressure, reduce their sexual activity. This means that earlier childbirth can be the result of those problems, not sexual intercourse. In 
addition, their study was retrospective, which would lead to biases in the results, because effective factors such as condom use, contact with semen, and seminal volume were not considered.

Omar et al. reported that intercourse during pregnancy had no significant effect on gestational age at the time of delivery although the average gestational age was lower in case of intercourse (11). According to a cohort study, intercourse at term pregnancy is not associated with earlier delivery (16). Nonetheless, in their review studies, Winer and Mozurkewich et al. cited that there is not enough evidence to evaluate the efficacy of intercourse for induction of labor $(9,17)$.

In this study, the samples of intercourse without contact with semen was too low that statistical analysis was impossible and we could not give an opinion about the impact of intercourse without vaginal contact with semen on symptoms of labor. Nevertheless, we compared mothers who had no vaginal contact with semen, including those who had no intercourse at all, with mothers who had such a contact. In this case, the results indicated that the mean gestational age was decrease significantly in case of vaginal contact with semen based on both LMP and ultrasonography. It is reported that semen provides the ripening of cervix because it contains prostaglandins, citric acid, fibrinolysin, and hyaluronidase $(3,15)$. Sekhavat et al. mentioned that prostaglandins concentration in the cervical mucus is 10- to 50-fold higher than normal values at two hours after intercourse. This cross-sectional study showed that sexual intercourse at term decreases prolonged pregnancy and induction of labor, and stimulates the onset of labor (4).

This study showed that gestational age based on LMP was significantly decreased with intercourse. In addition, gestational age was lower based on ultrasonography in women with intercourse during the final week of gestation. Therefore, in the absence of complications in pregnant women at term, sexual activity can be considered as a natural way to prevent post term pregnancy.

To encourage subjects to express their true feelings about their sexual activity, they were assured that their information would be remained confidential; however, some people were not able to mention their sexuality since sex is one of the most private affairs and because of cultural and religious restrictions. Then, lack of cooperation by individuals in expressing their concerns was researcher's limitation.

\section{Acknowledgements}

We thank Research Deputy of Kashan University of Medical Sciences that approved and financed this study.

\section{Authors 'Contributions}

Study concept and design: Mahboobeh Kafaei Atrian. Acquisition of data: Zohre Sadat. Analysis and interpretation of data and study supervision: Fatemeh Abbaszadeh. Drafting the manuscript: Mahbobeh Rasolzadeh Bidgoly. Statistical analysis: Mohammad Asghari Jafarabadi.

\section{Funding/Support}

The project was registered in Kashan University of Medical Sciences (No: 9057). Research Deputy of Kashan University of Medical Sciences approved and financed this study.

\section{References}

1. Leite AP, Campos AA, Dias AR, Amed AM, De Souza E, Camano L. Prevalence of sexual dysfunction during pregnancy. Rev Assoc Med Bras. 2009;55(5):563-8.

2. Babazadeh R, Najmabadi KM, Masomi Z. Changes in sexual desire and activity during pregnancy among women in Shahroud, Iran. Int J Gynaecol Obstet. 2013;120(1):82-4.

3. Kavanagh J, Kelly AJ, Thomas J. Sexual intercourse for cervical ripening and induction of labour. Cochrane Database Syst Rev. 2001(2):CD003093.

4. Sekhavat L, Akhavan Karbasi S. Effect of coital activity on hastening onset of labor and prevention of postdate pregnancy. Iran J Obstet Gynecol Infertil. 2010;13(2):13-6.

5. Tan PC, Andi A, Azmi N, Noraihan MN. Effect of coitus at term on length of gestation, induction of labor, and mode of delivery. $\mathrm{Ob}$ stet Gynecol. 2006;108(1):134-40.

6. Foumane P, Mboudou ET, Sama JD, Baba S, Enama Mbatsogo BA, Ngwana L. Sexual activity during pregnancy and prognosis of labor in Cameroonian women: a cohort study. J Matern Fetal Neonatal Med. 2014;27(13):1305-8.

7. Tan PC, Yow CM, Omar SZ. Effect of coital activity on onset of labor in women scheduled for labor induction: a randomized controlled trial. Obstet Gynecol. 2007;110(4):820-6.

8. Tan PC, Yow CM, Omar SZ. Coitus and orgasm at term: effect on spontaneous labour and pregnancy outcome. Singapore Med J. 2009;50(11):1062-7.

9. Winer N. [Different methods for the induction of labour in postterm pregnancy]. J Gynecol Obstet Biol Reprod (Paris). 2011;40(8):796-811.

10. Schaffir J. Sexual intercourse at term and onset of labor. Obstet Gynecol. 2006;107(6):1310-4.

11. Omar NS, Tan PC, Sabir N, Yusop ES, Omar SZ. Coitus to expedite the onset of labour: a randomised trial. BJOG. 2013;120(3):338-45.

12. Bello FA, Olayemi O, Aimakhu CO, Adekunle AO. Effect of pregnancy and childbirth on sexuality of women in ibadan, Nigeria. ISRN Obstet Gynecol. 2011;2011:856586.

13. Fox NS, Gelber SE, Chasen ST. Physical and sexual activity during pregnancy and near delivery. $J$ Womens Health (Larchmt). 2008;17(9):1431-5.

14. Ngwenya S, Lindow SW. 24 hour rhythm in the timing of prelabour spontaneous rupture of membranes at term. Eur J Obstet Gynecol Reprod Biol. 2004;112(2):151-3.

15. Tenore JL. Methods for cervical ripening and induction of labor. Am Fam Physician. 2003;67(10):2123-8.

16. Intercourse doesn't hasten onset of labor. $J$ Fam Pract. 2006;55(9):757.

17. Mozurkewich EL, Chilimigras JL, Berman DR, Perni UC, Romero VC, King VJ, et al. Methods of induction of labour: a systematic review. BMC Pregnancy Childbirth. 2011;11:84. 